PROCEEDINGS OF THE AMERICAN MATHEMATICAL SOCIETY

Volume 127, Number 3, March 1999, Pages 829-833

S 0002-9939(99)04552-9

\title{
CLOSURES OF TOTALLY GEODESIC IMMERSIONS INTO LOCALLY SYMMETRIC SPACES OF NONCOMPACT TYPE
}

\author{
TRACY L. PAYNE
}

(Communicated by Christopher Croke)

\begin{abstract}
It is established that if $\mathcal{M}_{1}$ and $\mathcal{M}_{2}$ are connected locally symmetric spaces of noncompact type where $\mathcal{M}_{2}$ has finite volume, and $\phi: \mathcal{M}_{1} \rightarrow \mathcal{M}_{2}$ is a totally geodesic immersion, then the closure of $\phi\left(\mathcal{M}_{1}\right)$ in $\mathcal{M}_{2}$ is an immersed "algebraic" submanifold. It is also shown that if in addition, the real ranks of $\mathcal{M}_{1}$ and $\mathcal{M}_{2}$ are equal, then the the closure of $\phi\left(\mathcal{M}_{1}\right)$ in $\mathcal{M}_{2}$ is a totally geodesic submanifold of $\mathcal{M}_{2}$. The proof is a straightforward application of Ratner's Theorem combined with the structure theory of symmetric spaces.
\end{abstract}

\section{INTRODUCTION}

In this paper, we consider totally geodesic maps and totally geodesic submanifolds. Let $\mathcal{D}$ and $\mathcal{M}$ be Riemannian manifolds. An immersion $\phi$ from $\mathcal{D}$ to $\mathcal{M}$ is called totally geodesic if for every geodesic $\gamma$ in $\mathcal{D}$, its image $\phi \circ \gamma$ is a geodesic in $\mathcal{M}$. A submanifold $\mathcal{N}$ of a Riemannian manifold $\mathcal{M}$ is called totally geodesic if the inclusion map $i: \mathcal{N} \rightarrow \mathcal{M}$ is a totally geodesic map.

Let $\mathcal{M}_{2}$ be a locally symmetric space of noncompact type, and let $G_{2}$ be the connected component of the identity in the isometry group of its universal cover $\widetilde{\mathcal{M}}_{2}$. We let $G_{2}$ act on $\mathcal{M}_{2}$ from the right. Let $p: \widetilde{\mathcal{M}}_{2} \rightarrow \mathcal{M}_{2}$ be the covering map.

We will prove

Theorem 1.1. Let $\mathcal{M}_{2}$ be a connected locally symmetric space of noncompact type with finite volume. Let $\mathcal{M}_{1}$ be a connected locally symmetric space of noncompact type. Let $\phi$ be a totally geodesic immersion of $\mathcal{M}_{1}$ into $\mathcal{M}_{2}$. Then the closure in $\mathcal{M}_{2}$ of the set $\phi\left(\mathcal{M}_{1}\right)$ is an immersed submanifold of $\mathcal{M}_{2}$ of the form $p(\tilde{x} H)$, where $\tilde{x}$ is a point in $\widetilde{\mathcal{M}}_{2}$ and $\tilde{x} H$ is the orbit of $\tilde{x}$ under a subgroup $H$ of $G_{2}$. If in addition, the rank of $\mathcal{M}_{1}$ is equal to the rank of $\mathcal{M}_{2}$, then the closure of $\phi\left(\mathcal{M}_{1}\right)$ is a totally geodesic submanifold of $\mathcal{M}_{2}$.

Furthermore, a result due to N. Shah [9], describes the algebraic structure of the subgroup $H$ : if $\mathcal{M}_{2}$ is either rank one or compact, then $H$ must be a reductive group with compact center.

In [10], A. Zeghib demonstrated that in a compact manifold with variable negative curvature, every immersed geodesic hypersurface of dimension two or more is

Received by the editors November 4, 1996 and, in revised form, June 10, 1997.

1991 Mathematics Subject Classification. Primary 53C42.

Key words and phrases. Totally geodesic, symmetric space, Ratner's Theorem.

(C)1999 American Mathematical Society 
compact, and that there are but a finite number of these objects. In the paper [8], N. Shah proved Theorem 1.1 in the case that $\mathcal{M}_{2}$ is a compact manifold of dimension $n>2$ with constant negative curvature and $\mathcal{M}_{1}$ is equal to $(n-1)$-dimensional hyperbolic space $\mathcal{H}^{n}$. Shah has informed the author that he also knew proofs for the finite volume and higher codimension cases. After the announcement of M. Ratner's powerful results describing the closures of unipotent orbits in quotients of Lie groups, Shah observed that his theorem followed from Ratner's Theorem and that the compactness hypothesis could be weakened to finite volume [2].

1.1. Acknowledgments. I would like to thank M. Ratner and R. Spatzier for their valuable suggestions and remarks. Special thanks are due to Nimish Shah, who showed how to simultaneously simplify the proof of the theorem and to drop a compactness hypothesis on $\mathcal{M}_{2}$ in the conclusion about totally geodesic submanifolds.

1.2. Notation and terminology. We will use capital letters $G, H$, etc. to denote Lie groups, and we will denote the corresponding Lie algebras by the corresponding gothic letters $\mathfrak{g}, \mathfrak{h}$, etc. If $\mathfrak{h}$ is a subalgebra of the Lie algebra $\mathfrak{g}, H$ will denote the connected subgroup of $G$ corresponding to $\mathfrak{h}$. For an element $g$ of $G$, we will use $c_{g}$ to denote conjugation by $g$. The terms "Cartan subalgebra" and "rank" will always mean an $\mathbf{R}$-split Cartan subalgebra and real rank.

\section{RATNER's ThEOREM}

Let $G$ be a second countable real Lie group. Suppose $\Gamma$ is a lattice in $G$. The group $G$ acts on the quotient $G / \Gamma$. A subset $A$ of $G / \Gamma$ is called homogeneous if there is a closed subgroup $H$ of $G$ and a point $x$ in $G / \Gamma$ such that $A$ is the orbit $H x$ of $H$ through $x$ and $H \cap g \Gamma g^{-1}$ is a lattice in $H$, where $g x \in A$.

For $g$ in $G$, let $\operatorname{Ad}_{g}: \mathfrak{g} \rightarrow \mathfrak{g}$ denote the adjoint map of $g$ defined by $\operatorname{Ad}_{g}(X)=$ $d c_{g}(X)$ for $X$ in $\mathfrak{g}$. Let ad $: \mathfrak{g} \rightarrow \operatorname{End}(\mathfrak{g})$ denote the differential of Ad $: G \rightarrow G L(\mathfrak{g})$. An element $u$ of $G$ is called unipotent if $\operatorname{Ad}_{u}-I$ is nilpotent. A subgroup of $G$ is unipotent if all its elements are unipotent.

Let $U$ be a unipotent subgroup of $G$. In a series of articles [3], [4], [5], [6], M. Ratner has completely analyzed the closures of orbits $U x$ of unipotent subgroups and classified the ergodic $U$-invariant Borel probability measures on $G / \Gamma$. See [7] for a survey. We will use the following very powerful theorem of M. Ratner on orbit closures of orbits of unipotent groups.

Theorem 2.1 (M. Ratner [6]). Let $G$ be a connected Lie group and $U$ a connected subgroup of $G$ generated by unipotent elements of $G$. Then, for any lattice $\Gamma$ in $G$ and any $x$ in $G / \Gamma$, the closure of the orbit $U x$ in $G / \Gamma$ is homogeneous.

An element of $X$ of $\mathfrak{g}$ is called diagonal for a nilpotent element $Y$ of $\mathfrak{g}$ if there is a nilpotent element $Y^{*} \in \mathfrak{g}$ such that $\operatorname{ad}\left(Y^{*}\right)(Y)=X, \operatorname{ad}(X)(Y)=-2 Y$ and $\operatorname{ad}(X)\left(Y^{*}\right)=2 Y^{*}$. The elements $Y$ and $Y^{*}$ generate a subalgebra of $\mathfrak{g}$ isomorphic to $\mathfrak{s l}(2, \mathbf{R})$ so that $X$ is mapped to $\operatorname{diag}(1,-1)$. The one-parameter subgroup $A=\{\exp (t X) \mid t \in \mathbf{R}\}$ of $G$ is called diagonal for the one-parameter unipotent subgroup $U=\{\exp (t Y) \mid t \in \mathbf{R}\}$ of $G$. Given $A$ diagonal for $U$, we will denote by $S L_{2}(U, A)$ the subgroup generated by $A=\{\exp t X\}_{t \in \mathbf{R}}, U=\{\exp t Y\}_{t \in \mathbf{R}}$ and $U^{*}=\left\{\exp t Y^{*}\right\}_{t \in \mathbf{R}}$. 
The next lemma follows from Proposition 2.1 in [5]. It will be used in the part of the proof of Theorem 1.1 that shows that the submanifold is totally geodesic in certain cases.

Proposition 2.2 (M. Ratner). Let $G$ be a connected Lie group and $U$ a connected subgroup generated by unipotent elements of $G$. Let $A_{1}, \ldots, A_{n}$ be diagonal for some one-parameter subgroups $U_{1}, \ldots, U_{n}$ of $U$. Let $L$ be the subgroup generated by $U$ and $A_{1}, \ldots, A_{n}$, and let $L^{\prime}$ be the subgroup generated by $U$ and $S L_{2}\left(U_{i}, A_{i}\right)$. Then $\overline{L x}=\overline{L^{\prime} x}$ for all $x \in G / \Gamma$.

\section{Globally symmetric spaces AND THEIR TOTALLY GEODESIC SUBMANIFOLDS}

Let $\mathcal{M}$ be a globally symmetric space of noncompact type, and let $G$ be the connected component of the identity in its isometry group. Fix a point $p$ in $\mathcal{M}$. Then $\mathcal{M}$ can be identified with the homogeneous space $K \backslash G$, where $K$ is the stabilizer in $G$ of the point $p$. The group $G$ is a semisimple Lie group of noncompact type, and $K$ is a maximal compact subgroup of $G$. The point $p$ induces a Cartan decomposition $\mathfrak{g}=\mathfrak{k}+\mathfrak{p}$ of $\mathfrak{g}$ and a corresponding Cartan involution. We can then identify $T_{p} \mathcal{M}$ with $\mathfrak{p}$.

Using this set-up, the next theorem from [1] gives an algebraic description of totally geodesic submanifolds.

Theorem 3.1. Suppose that $\mathcal{N}$ is a totally geodesic submanifold of $\mathcal{M}$ which contains the point $p$.

Then $\mathcal{N}$ is a globally symmetric space. Let $\mathfrak{p}^{*}$ be the subspace of $\mathfrak{p}$ which is identified with $T_{p} \mathcal{N}$ under the identification of $T_{p} \mathcal{N}$ and $\mathfrak{p}$. Let $\mathfrak{k}^{*}=\left[\mathfrak{p}^{*}, \mathfrak{p}^{*}\right]$ and let $\mathfrak{g}^{*}=\mathfrak{k}^{*}+\mathfrak{p}^{*}$. Let $G^{*}$ and $K^{*}$ be the corresponding subgroups of $G$. The submanifold $\mathcal{N}$ has the structure of $G^{*} / K^{*}$ and the corresponding Cartan decomposition relative to the point $p$ is $\mathfrak{g}^{*}=\mathfrak{k}^{*}+\mathfrak{p}^{*}$, with the Cartan involution for $\mathfrak{g}^{*}$ equal to the restriction of the Cartan involution for $\mathfrak{g}$ to $\mathfrak{g}^{*}$.

Conversely, if $\mathfrak{g}^{*}$ is a subalgebra of $\mathfrak{g}$ which is invariant under the Cartan involution for $\mathfrak{g}$, then it gives rise to a totally geodesic submanifold $\mathcal{N}$ of $\mathcal{M}$. Let $\mathfrak{p}^{*}=\mathfrak{g}^{*} \cap \mathfrak{p}$. Then

$$
\mathcal{N}=\left\{\exp (X) K \mid X \in \mathfrak{p}^{*}\right\}
$$

is a totally geodesic submanifold of $\mathcal{M}$.

\section{Proof of Theorem 1.1}

First, note that we may assume that $\mathcal{M}_{1}$ is simply connected. If it is not, let $\rho_{1}$ be a locally isometric covering map of $\mathcal{M}_{1}$ by its universal cover $\widetilde{\mathcal{M}}_{1}$. Then the map $\phi \circ \rho_{1}$ is a totally geodesic immersion of $\widetilde{\mathcal{M}}_{1}$ into $\mathcal{M}_{2}$ so that the closure of $\left(\phi \circ \rho_{1}\right)\left(\overline{\mathcal{M}_{1}}\right)$ is equal to the closure of $\phi\left(\mathcal{M}_{1}\right)$.

Now, we find algebraic descriptions of $\mathcal{M}_{1}$ and $\mathcal{M}_{2}$ that are compatible through the map $\phi$. Let $\rho$ be a locally isometric covering map of $\mathcal{M}_{2}$ by its universal cover $\widetilde{\mathcal{M}}_{2}$. The map $\phi$ lifts to a one-to-one map $\tilde{\phi}: \mathcal{M}_{1} \rightarrow \widetilde{\mathcal{M}}_{2}$ such that $\rho \circ \tilde{\phi}=\phi$. Note that $\tilde{\phi}$ is also a totally geodesic isometric immersion, and the submanifold $\tilde{\phi}\left(\mathcal{M}_{1}\right)$ is a totally geodesic submanifold of $\widetilde{\mathcal{M}}_{2}$. Let $\tilde{x}$ be a point in $\tilde{\phi}\left(\mathcal{M}_{1}\right)$ and let $x=\rho(\tilde{x})$.

Since the map $\phi$ is totally geodesic, the geodesic symmetries are preserved under $\phi$. Since the geodesic symmetries determine the algebraic structure of $G_{1}$ and $G_{2}$, we 
may assume, by rescaling the metric on irreducible components of $\mathcal{M}_{1}$ if necessary, that the map $\phi$ is a local isometry.

Let $G_{2}$ denote the connected component of the identity in the isometry group of $\widetilde{\mathcal{M}}_{2}$. The globally symmetric space $\widetilde{\mathcal{M}}_{2}$ has the structure of $K_{2} \backslash G_{2}$, where $K_{2}$ is the stabilizer of the point $\tilde{x}$ in $G_{2}$. The manifold $\mathcal{M}_{2}$ then has the structure of $K_{2} \backslash G_{2} / \Gamma$, where $\Gamma$ is the group of deck transformations for the covering map $\rho$. Let $p$ denote the covering map $p: G_{2} / \Gamma \rightarrow K_{2} \backslash G_{2} / \Gamma$. Let $\mathfrak{g}_{2}=\mathfrak{k}_{2}+\mathfrak{p}_{2}$ be the Cartan decomposition of $\mathfrak{g}_{2}$ induced by $\tilde{x}$ and let $\theta$ be the corresponding Cartan involution.

By Theorem 3.1, the submanifold $\tilde{\phi}\left(\mathcal{M}_{1}\right)$ is globally symmetric. Let $G_{1}$ be the subgroup of $G_{2}$ that is the connected component of the identity in the isometry group of $\tilde{\phi}\left(\mathcal{M}_{1}\right)$. Then $\tilde{\phi}\left(\mathcal{M}_{1}\right)$ is the orbit $\tilde{x} G_{1}$.

Let $\hat{x}$ be the identity coset of $G_{2} / \Gamma$, so that $p(\hat{x})=x$ and $\phi\left(\mathcal{M}_{1}\right)=p\left(G_{1} \hat{x}\right)$. If $G$ is a connected semisimple Lie group of noncompact type, then a semisimple subgroup of $G$ without compact factors is generated by unipotent elements of $G$, so $G_{1}$ is generated by unipotent elements. Then by Ratner's Theorem 2.1, the closure of the orbit $G_{1} \hat{x}$ in $G_{2} / \Gamma$ is homogeneous. There is a closed subgroup $H$ of $G_{2}$ such that the closure of $G_{1} \hat{x}$ equals the orbit $H \hat{x}$ and $H \cap \Gamma$ is a lattice in $H$.

The projection $p(H \hat{x})$ of the homogeneous set $H \hat{x}$ equals $\overline{\phi\left(\mathcal{M}_{1}\right)}$. Since the kernel of $p$ is compact, if $\mathcal{A}$ is a set contained in $G / \Gamma$, then $p(\overline{\mathcal{A}})=\overline{p(\mathcal{A})}$. Thus,

$$
\overline{\phi\left(\mathcal{M}_{1}\right)}=\overline{p\left(G_{1} \hat{x}\right)}=p\left(\overline{G_{1} \hat{x}}\right)=p(H \hat{x}) .
$$

Since $\Gamma \cap H$ is a lattice in $H$ and $\Gamma$ has no torsion, the set $H \hat{x}$ is a submanifold of $G_{2} / \Gamma$ with the structure of $H / \Gamma \cap H$. Then the set $p(H \hat{x})$ is a submanifold of $\mathcal{M}_{2}$ with the structure of $K_{2} \cap H \backslash H / \Gamma \cap H$.

Now we would like to show that if the ranks of $\mathcal{M}_{1}$ and $\mathcal{M}_{2}$ are equal, then the subset $p(H \hat{x})$ of $\mathcal{M}_{2}$ is a totally geodesic submanifold.

Let $\mathfrak{a}$ be a Cartan subalgebra for $\mathfrak{g}_{1}$ contained in $\mathfrak{p}_{1}$. Since the ranks of $\mathcal{M}_{1}$ and $\mathcal{M}_{2}$ are equal, $\mathfrak{a}$ is a Cartan subalgebra for $\mathfrak{g}_{2}$. Let

$$
\mathfrak{g}_{2}=\mathfrak{g}_{0}^{2}+\sum_{\beta \in \Lambda_{2}} \mathfrak{g}_{\beta}
$$

be the corresponding root space decomposition. Because $\mathfrak{g}_{1}<\mathfrak{h}$, we know that $\mathfrak{a}<\mathfrak{h}$. The Cartan decomposition for $\mathfrak{g}_{2}$ restricts to a Cartan decomposition for $\mathfrak{h}$ :

$$
\mathfrak{h}=\mathfrak{h}_{0} \oplus \sum_{\alpha \in \Lambda} \mathfrak{h}_{\alpha}
$$

Note that for all $\alpha \in \Lambda$, the root space $\mathfrak{h}_{\alpha}$ is contained in a root space $\mathfrak{g}_{\beta}$ for some $\beta$ in $\Lambda_{2}$.

The subalgebra $\mathfrak{k}_{2}$ of $\mathfrak{g}_{2}$ is pointwise fixed under $\theta$ and all one-dimensional subspaces of $\mathfrak{a}$ are fixed under $\theta$, and hence any subspace of $\mathfrak{k}_{2} \oplus \mathfrak{a}$ is preserved under $\theta$. Since $\mathfrak{h}_{0}$ is contained in $\mathfrak{g}_{2}^{0}=\left(\mathfrak{k}_{2} \cap \mathfrak{g}_{2}^{0}\right) \oplus \mathfrak{a}, \mathfrak{h}_{0}$ is invariant under $\theta$.

Now suppose that $\mathfrak{b}_{\alpha}$ is a one-dimensional subspace of $\mathfrak{h}_{\alpha}$ for some $\alpha \in \Lambda$. The subalgebra $\mathfrak{s l}_{\alpha}$ of $\mathfrak{g}_{2}$ spanned by the one-dimensional subspaces $\mathfrak{b}_{\alpha}, \theta\left(\mathfrak{b}_{\alpha}\right)$ and $\left[\mathfrak{b}_{\alpha}, \theta\left(\mathfrak{b}_{\alpha}\right)\right]$ is isomorphic to $\mathfrak{s l}(2, \mathbf{R})$, and $\left[\mathfrak{b}_{\alpha}, \theta\left(\mathfrak{b}_{\alpha}\right)\right]<\mathfrak{a}$. Since $\mathfrak{b}_{\alpha} \oplus\left[\mathfrak{b}_{\alpha}, \theta\left(\mathfrak{b}_{\alpha}\right)\right]<$ $\mathfrak{h}$, by Proposition $2.2, \mathfrak{s l}_{\alpha}<\mathfrak{h}$. Letting $\alpha$ vary over $\Lambda$, we see that $\sum_{\alpha \in \Lambda} \mathfrak{h}_{\alpha}$ is invariant under $\theta$. Already knowing that $\mathfrak{h}_{0}$ is invariant under $\theta$, we conclude that $\mathfrak{h}$ is invariant under $\theta$. 
By Theorem 3.1, the orbit $H \hat{x}$ in $G_{2} / \Gamma$ projects to a totally geodesic submanifold of $K_{2} \backslash G_{2} / \Gamma$. Thus, $\overline{\phi\left(\mathcal{M}_{1}\right)}=p(H \hat{x})$ is a totally geodesic submanifold of $\mathcal{M}_{2}$, concluding the proof of Theorem 1.1.

\section{EXAMPLES}

The simplest example of the situation described in Theorem 1.1 is the case when the image of the totally geodesic immersion is closed.

Example 5.1. Let $\mathcal{M}_{2}$ be a locally symmetric space of noncompact type with finite volume, and let $\mathcal{M}_{1}$ be a closed totally geodesic submanifold of $\mathcal{M}_{2}$. Let $\rho: \widetilde{\mathcal{M}}_{1} \rightarrow \mathcal{M}_{1}$ be the locally isometric covering map of $\mathcal{M}_{1}$ by its universal cover. Let $i: \mathcal{M}_{1} \rightarrow \mathcal{M}_{2}$ be the inclusion map. Then the map $\phi=i \circ \rho$ is totally geodesic. The closure of $\phi\left(\widetilde{\mathcal{M}_{1}}\right)$ in $\mathcal{M}_{2}$ is $\mathcal{M}_{1}$.

It more often happens that the image of the totally geodesic immersion is not closed, as in the example below.

Example 5.2. Let $\mathcal{M}_{1}, \mathcal{M}_{2}$ and $\phi$ be as in Example 5.1. Let $\gamma$ be a geodesic in $\mathcal{M}_{1}$ which is dense in $\mathcal{M}_{1}$, and let $\tilde{\gamma}$ be the geodesic in $\widetilde{\mathcal{M}_{1}}$ that covers $\gamma$. Let $\mathcal{M}_{3}$ be any totally geodesic submanifold of $\overline{\mathcal{M}_{1}}$ such that $\tilde{\gamma}$ is contained in $\mathcal{M}_{3}$. The restriction of $\phi$ to $\mathcal{M}_{3}$ is a totally geodesic immersion from $\mathcal{M}_{3}$ to $\mathcal{M}_{2}$. The geodesic $\gamma$ is in its image. Then since

$$
\mathcal{M}_{1}=\overline{(\gamma)} \subset \overline{\left.\phi\right|_{\mathcal{M}_{3}}\left(\mathcal{M}_{3}\right)} \subset \mathcal{M}_{1},
$$

the closure of $\left.\phi\right|_{\mathcal{M}_{3}}\left(\mathcal{M}_{3}\right)$ in $\mathcal{M}_{2}$ is equal to $\mathcal{M}_{1}$.

\section{REFERENCES}

1. P. Eberlein, Geometry of nonpositively curved manifolds, Chicago Lectures in Mathematics, University of Chicago Press, Chicago, IL, 1996. CMP 97:10.

2. E. Ghys, Dynamique des flots unipotents sur les espaces homogènes, Astérisque 206 (1992), 93-136. MR 94e:58101

3. M. Ratner, On measure rigidity of unipotent subgroups of semisimple groups, Acta Math. 165 (1990), 229-309. MR 91m:57031

4. _ Strict measure rigidity for unipotent subgroups of solvable groups, Invent. Math. 101 (1990), 449-482. MR 92h:22015

5. _ On Raghunathan's measure conjecture, Ann. of Math. 134 (1991), 545-607. MR 93a:22009

6. L Raghunathan's topological conjecture and distributions of unipotent flows, Duke Math. J. 63 (1991), no. 1, 235-280. MR 93f:22012

7. Invariant measures and orbit closures for unipotent actions on homogeneous spaces, Geom. Funct. Anal. 4 (1994), no. 2, 236-257. MR 95c:22018

8. N. Shah, Closures of totally geodesic immersions in manifolds of constant negative curvature, Group Theory from a Geometrical Viewpoint, World Sci. Pub., 1991, pp. 718-732. MR 93d:53078

9. Uniformly distributed orbits of certain flows on homogeneous spaces, Math. Ann. 289 (1991), 315-334. MR 93d:22010

10. A. Zeghib, Laminations et hypersurfaces géodésiques des variétés hyperboliques, Ann. scient. Éc. Norm Sup. 24 (1991), 171-188. MR 92b:53098

Department of Mathematics, Washington University, St. Louis, Missouri 63130-4899

E-mail address: tpayne@math.wustl.edu France

Current address: École Normale Supérieure de Lyon, 46, Allée d'Italie, 69364 Lyon Cedex 07,

E-mail address: tpayne@umpa.ens-lyon.fr 